\title{
Minimally invasive and surface electroporation delivery of DNA vaccines for the induction of robust humoral immune responses against HIV antigens
}

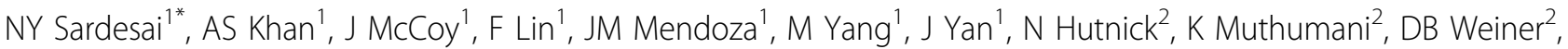 \\ KE Broderick ${ }^{1}$
}

From AIDS Vaccine 2012

Boston, MA, USA. 9-12 September 2012

\section{Background}

Clinical data from the HVTN-080 study demonstrated that intramuscular electroporation (EP) delivery of PENNVAX $^{\mathbb{B}}$-B DNA vaccine and the plasmid adjuvant IL-12 generated strong antigen specific cellular immune responses in humans with nearly $90 \%$ response rate. We have now developed minimally invasive EP delivery technologies (MID-EP) to target dermal tissue and demonstrate their ability to generate strong antibody (Ab) responses in animal models with DNA antigens including small pox, influenza, dengue - and have shown protection from viremia and lethality following challenge.

\section{Methods}

We demonstrate MID-EP delivery of consensus HIV gp140 antigens and show the generation of cross-clade neutralizing responses in guinea pigs and rabbits. These EP enhanced humoral responses were significantly broader and higher than naked DNA delivery alone or with a protein antigen. We demonstrated NAb titers against a broad panel of 15 Tier-1 HIV viruses from Clades A-D in the range of 20-200 measured in the Tzm-Bl neutralization assay. The magnitude but not the breadth of the responses was boosted to 20-1000 range using a MID-EP DNA prime-protein boost regimen.

\section{Results}

We further developed a surface EP device (SEP) for the simultaneous, but spatially segregated, delivery of multicomponent HIV vaccines. The SEP device operates under substantially lower voltage parameters than conventional EP devices resulting in significant improvements in tolerability. The separation of multi-component HIV vaccines avoids potential issues with plasmid interference at the transcriptional or translational levels. SEP produces $\mathrm{Ab}$ responses comparable to the penetrating DNAEP devices.

\section{Conclusion}

Our results suggest that MID/SEP electroporation devices offer safe, tolerable and potent methods to administer HIV DNA vaccinations in a prophylactic clinical setting. Combined with the design of novel HIV consensus based Env antigens these DNA-EP combination vaccines are suitable for further HIV vaccine product development.

\section{Author details}

${ }^{1}$ Inovio Pharmaceuticals, Blue Bell, PA, USA. ${ }^{2}$ University of Pennsylvania School of Medicine, Philadelphia, PA, USA.

Published: 13 September 2012

doi:10.1186/1742-4690-9-S2-O9

Cite this article as: Sardesai et al.: Minimally invasive and surface electroporation delivery of DNA vaccines for the induction of robust humoral immune responses against HIV antigens. Retrovirology 2012 9(Suppl 2):09.

Inovio Pharmaceuticals, Blue Bell, PA, USA

Full list of author information is available at the end of the article

(c) 2012 Sardesai et al; licensee BioMed Central Ltd. This is an Open Access article distributed under the terms of the Creative Commons 